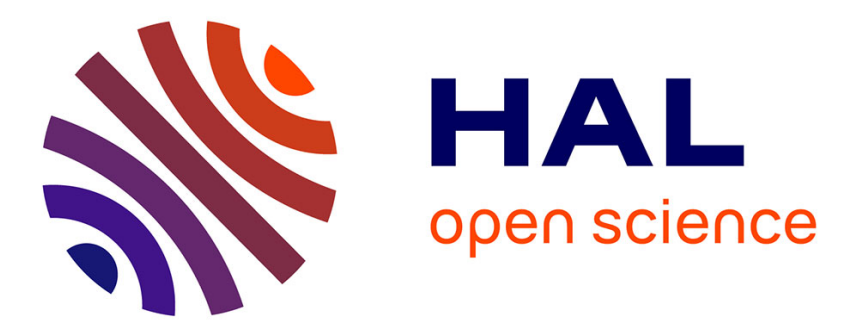

\title{
Noise evasion properties of electrostatic gap-closing MEMS resonators with pulsed excitation waveforms
}

\author{
Jérôme Juillard, Antonio Somma, Alexis Brenes
}

\section{To cite this version:}

Jérôme Juillard, Antonio Somma, Alexis Brenes. Noise evasion properties of electrostatic gap-closing MEMS resonators with pulsed excitation waveforms. Joint Conference of the IEEE International Frequency Control Symposium and IEEE International Symposium on Applications of Ferroelectrics (IFCS-ISAF 2020), Jul 2020, Online, United States. Paper ID 4424. hal-02962433

\section{HAL Id: hal-02962433 \\ https://hal.science/hal-02962433}

Submitted on 9 Oct 2020

HAL is a multi-disciplinary open access archive for the deposit and dissemination of scientific research documents, whether they are published or not. The documents may come from teaching and research institutions in France or abroad, or from public or private research centers.
L'archive ouverte pluridisciplinaire HAL, est destinée au dépôt et à la diffusion de documents scientifiques de niveau recherche, publiés ou non, émanant des établissements d'enseignement et de recherche français ou étrangers, des laboratoires publics ou privés. 


\title{
Noise evasion properties of electrostatic gap-closing MEMS resonators with pulsed excitation waveforms
}

\author{
Jérôme Juillard ${ }^{1}$, Antonio Somma ${ }^{1}$, Alexis Brenes ${ }^{2}$ \\ ${ }^{1}$ GEEPS (UMR8507, CNRS, CentraleSupélec, Université Paris-Saclay, Sorbonne Université), Gif-sur-Yvette, France \\ ${ }^{2}$ LISITE, ISEP, Paris, France \\ jerome.juillard@centralesupelec.fr
}

\begin{abstract}
The limits on MEMS resonant sensor performance set by nonlinearity are often studied through a model of a Duffing resonator, with linear actuation. This model largely fails to capture the properties of MEMS resonant sensors with electrostatic gap-closing actuation. We have shown that a specific feature of such ubiquitous resonators is that their stability is strongly sensitive to the waveform used to drive them to resonance. In this paper, we conduct an analytical investigation of these phenomena and validate our theoretical results transient simulations.
\end{abstract}

Keywords—resonant sensors, frequency stability, nonlinearity, electrostatic actuation.

\section{INTRODUCTION}

In many respects, nonlinearity limits the performance of resonant sensors. In the MEMS domain [1-5], this is often studied through a model of a weakly-nonlinear (Duffing) resonator, with linear actuation where actuation force $F_{\text {act }}$, which sets the resonator in motion, is proportional to the excitation voltage $V_{\text {exc }}$ delivered by the electronic feedback loop. In that framework, the accuracy of a resonator depends on only two adjustable parameters: the amplitude $V$ and the phase $\phi$ of the $1^{\text {st }}$ harmonic of $V_{\text {exc }}$. When $\phi=90^{\circ}$, which is nominal for drive efficiency, the best accuracy is obtained at a critical drive amplitude $V_{\text {crit }}$ above which it degrades, through nonlinearity [1-2].

The Duffing/linear actuation (DLA) model is handy and serves as the starting point for several inspiring studies. For example, in [4], the authors propose a passive temperaturecompensation scheme for comb-driven MEMS resonators. Their approach is to adjust $V$ so that temperature-induced variations of $Q$ and natural frequency $\omega_{0}$ compensate through the A-f effect [2], leaving $\phi$ to the value of $90^{\circ}$. While temperature sensitivity is quantitatively reduced, the proposed nonlinear operation point results in poorer noise performance $\left(V>V_{\text {crit }}\right)$. In [5], the authors use piezoelectrically-driven MEMS resonators to explore the various oscillation regimes predicted by the DLA model. They verify the existence of parametric evasion regimes, where oscillation frequency $\omega$ becomes insensitive to certain parametric fluctuations (e.g. fluctuations of the feedback phase), independently of quality factor. However, these correspond to $\phi \neq 90^{\circ}$ and can only be reached at the cost of reduced drive efficiency.

While the DLA model applies to electrostatic comb drives and piezoelectric drives, it fails to capture the more complex properties of electrostatic gap-closing actuators. This fact is largely overlooked by most studies in the field, even though electrostatic gap-closing actuation is very commonplace. The studies conducted in [4-5] should then be reexamined in this framework.
In this paper, we conduct a study of parametric evasion properties of MEMS oscillators using an electrostatic gapclosing actuation (EGCA) scheme. This study is based on a simplified analytical model, using a first harmonic, slowlyvarying amplitude and phase approximation, as described in section II. It highlights that waveform-dependent feedback phase evasion, predicted by a quasistatic model in [6-7], is limited to the Leeson frequency bandwidth of the oscillator. Transient simulation is then used in section III to support this result and verify the validity of our first-harmonic approximation. Section IV presents some conclusions and perspectives.

\section{ANALYSIS}

\section{A. Framework}

We consider a simplified model of a MEMS oscillator, consisting of a one-port MEMS resonator with nonlinear mechanical stiffness (e.g. nonlinearity resulting from stressstiffening) and a parallel-plate EGCA scheme, and of some idealized feedback electronics, through which a voltage can be applied to the resonator. This voltage is the sum of a DC bias $V_{\text {bias }}$ and of a drive component $V_{\text {drive }} \ll V_{\text {bias }}$. This is represented in Fig. 1. The mechanical motion of the resonator is translated into an electrical signal through a transduction scheme, which is used to generate the drive component of the actuation voltage. $V_{\text {drive }}$ is then converted into a force through EGCA. The first harmonic of the driving force should be leading that of the mechanical motion of the resonator by $90^{\circ}$ to maximize drive efficiency and operate at resonance. The dynamics of the oscillator are captured in the following nondimensional model

$$
\left(1+\gamma x^{2}-\frac{\eta}{(1-x)^{2}}\right) x+\frac{\dot{x}}{Q}+\ddot{x}=\frac{2 \eta}{(1-x)^{2}} v(t)
$$

where $x$ is the mechanical motion of the resonator $(x=1$ means the resonator is in contact with the facing electrode), $t$ is time (nondimensionalized so that the natural period of the resonator is $2 \pi), v \ll 1$ is the ratio of $V_{\text {drive }}$ to $V_{\text {bias }}, Q \gg 1$ is the resonator quality factor, $\gamma>0$ accounts for mechanical stiffening effects, $\eta>0$ is an electromechanical transduction coefficient (proportional to $V_{\text {bias }}^{2}$ ) and a dot represents differentiation with respect to $t$. In what follows, we study the cases when the drive voltage consists either of short positive voltage pulses with duration $\tau \ll 2 \pi$, delivered on rising zerocrossings of $x$, or of negative pulses delivered on falling zerocrossings of $x$ (Fig. 2). Because of the dependence on $x$ of the multiplicative coefficient on the right-hand side of (1), these two driving schemes have fundamentally different properties with respect to frequency stability. On the other hand, they are both immune to resonant pull-in [8-9] provided $Q \gg 1$ and 


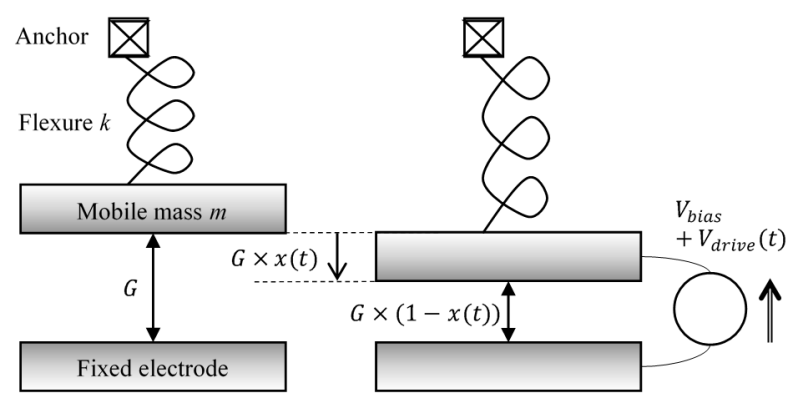

Fig. 1. Resonator with parallel-plate electrostatic gap-closing actuation.

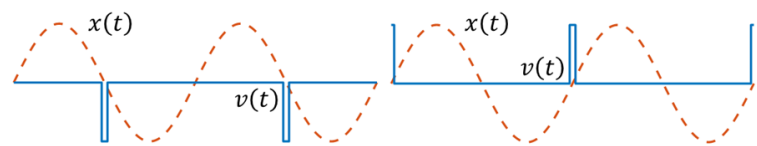

Fig. 2. Negative pulse drive (left) and positive pulse drive (right).

$\eta \ll 4 / 27$ (this limiting value corresponds to static pull-in), meaning that they can be used to make the resonator oscillate at an arbitrary amplitude without risk. In what follows, we study the impact on frequency stability of fluctuations of the feedback phase $\delta \phi$ from the nominal $90^{\circ}$ value.

\section{B. Slowly-varying first harmonic perturbation model}

Let us suppose $x=(X+\delta X) \sin ((\omega+\delta \omega) t)$, where $\delta X$ and $\delta \omega$ are slowly-varying fluctuations of the steady-state amplitude $X<1$ and frequency $\omega \approx 1$. Harmonic balance lets us split the governing dynamics into quadrature and phase components, to which first-order perturbation can be applied. The steady-state solution is

$$
\begin{gathered}
X=2 \eta Q V=Q F \\
\omega=\sqrt{1+\frac{3}{4} \gamma X^{2}-\frac{2 \eta}{\left(1-X^{2}\right)^{\frac{3}{2}}}} \approx 1+\frac{1}{2} g(X)
\end{gathered}
$$

where $V$ is the amplitude of the first harmonic of $v$ [6], and $F$ that of the (nondimensional) driving force. The fluctuations $\delta X$ and $\delta \omega$ are governed by

$$
\begin{gathered}
\delta \dot{X}+\frac{1}{2 Q} \delta X= \pm F X \delta \phi \\
\delta \omega=\frac{1}{2 Q} \delta \phi+\frac{1}{2} g^{\prime}(X) \delta X
\end{gathered}
$$

where a prime denotes differentiation with respect to $X$, and the sign on the right-hand side of (4) depends on the polarity of the pulses ( + corresponds to the negative pulse case). For better insight, (4) means that, in an EGCA oscillator with positive or negative pulse drive, amplitude responds as a firstorder lowpass system to phase fluctuations. This is as opposed to DLA oscillators, whose amplitude is independent of feedback phase (provided $\phi=90^{\circ}$ ), or to EGCA oscillators with other driving waveforms. According to (5), these amplitude fluctuations are then transformed into frequency fluctuations through the second-term on the right-hand side, resulting from static nonlinearity. It is straightforward to simplify (4-5) into a single equation to obtain the transfer function governing the conversion of feedback phase fluctuations into frequency fluctuations:

$$
\frac{\delta \omega}{\delta \phi}\left(j \omega_{\phi}\right)=\frac{1}{2 Q} \times \frac{j \omega_{\phi}+\frac{C(X)}{2 Q}}{j \omega_{\phi}+\frac{1}{2 Q}}
$$

where $\omega_{\phi}$ is the frequency of the feedback phase fluctuations and

$$
C(X)=1 \pm 2 Q g^{\prime}(X) X^{2}
$$

\section{Qualitative analysis}

In the case of resonators with no mechanical hardening $(\gamma=0),(7)$ becomes

$$
C(X)=1 \mp 12 \eta Q X^{3}\left(1-X^{2}\right)^{-\frac{5}{2}}
$$

We see that $C \approx 1$ for small values of $X$ and monotonically goes to $\mp \infty$ as $X$ goes to 1 . This implies the existence of an optimal operating amplitude between $X=0$ and 1 , where $C=0$, when negative pulses are used. At this amplitude, evasion from feedback phase fluctuations is achieved, in the sense that oscillation frequency is completely immune to quasistatic fluctuations of $\phi$. When mechanical hardening is also present, the same scenario prevails provided $\gamma<4 \eta$. Above this threshold, $g$ is increasing between 0 and

$$
X_{\text {lin }}=\left(1-\left(\frac{4 \eta}{\gamma}\right)^{\frac{2}{5}}\right)^{\frac{1}{2}}
$$

decreasing between $X_{\text {lin }}$ and 1 . Consequently, there are at least one, and up to two evasion points, between 0 and $X_{\text {lin }}$ when positive pulses are used. When negative pulses are used, there is a single evasion point (between $X_{\text {lin }}$ and 1).

Regardless of nonlinearity, we also have the following properties. If $C=0$ for one of the waveforms, then $C=2$ for the other, meaning the sensitivity to quasistatic $\left(\omega_{\phi} \approx 0\right)$ feedback phase fluctuations is twice as large as in the linear case. At the Leeson frequency $\left(\omega_{\phi}=1 / 2 Q\right)$, the sensitivity to feedback phase fluctuations is $\sqrt{2} \approx 1.4$ times smaller than in the linear case if $C=0$ (i.e. at an evasion point), and $\sqrt{5 / 2} \approx 1.6$ times greater if $C=2$.

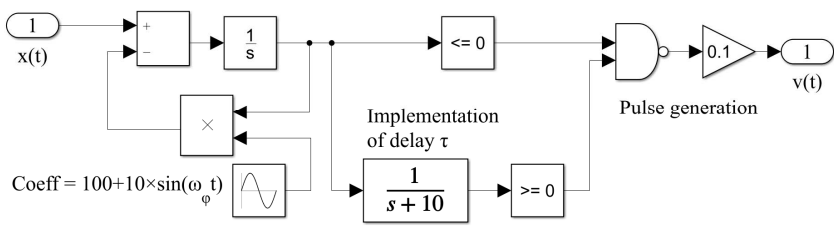

Fig. 3. System-level implementation of negative pulse drive in Simulink.

\section{VALIDATION}

\section{A. Simulation framework}

Transient simulations of (1) are performed with Matlab/Simulink. Feedback phase is modulated by harmonically varying, with frequency $\omega_{\phi}$, the cutoff frequency of a lowpass filter in the oscillator loop. Pulses of duration $\tau$ are generated on the zero-crossings of the output of this filter, nearly in phase with $x$, by performing a logical comparison between the sign of this signal and that of a phase- 
shifted version of this signal. $V$ is then set by changing the gain at the output of this chain, as shown in Fig. 3. For each simulation, we set the value of $V$ and of $\omega_{\phi}$ and choose whether positive or negative pulses are used. Two sets of simulations are performed: one in which the resonator has a purely softening characteristic $\left(\eta=5 \times 10^{-3}, \gamma=0\right)$, another with a mixed softening-hardening characteristic $(\eta=$ $\left.5 \times 10^{-3}, \gamma=8 \eta\right)$. The resonator's $Q$ is 1000 . The instantaneous oscillation period can be precisely evaluated by calculating the simulation time elapsed between two successive zero-crossings of $x$. Gear's method (ode15s) is used as numerical solver; all parameters are set to their default values, except RelTol and AbsTol that are both set to $10^{-8}$.

\section{B. Results}

Simulation results are shown in Fig. 4, 5, 6, 7. For comparison, we have also plotted the results expected from the analytical formula (6) in the case $\omega_{\phi} \approx 0$ and $\omega_{\phi}=1 / 2 Q$. We can see that the results behave as expected from the qualitative analysis and, on the whole, are in excellent quantitative agreement with the analytical model except in the case of negative pulses with mechanical hardening, for which the agreement is not as good. The reason why this simulation in particular should agree less with theory remains to be found.

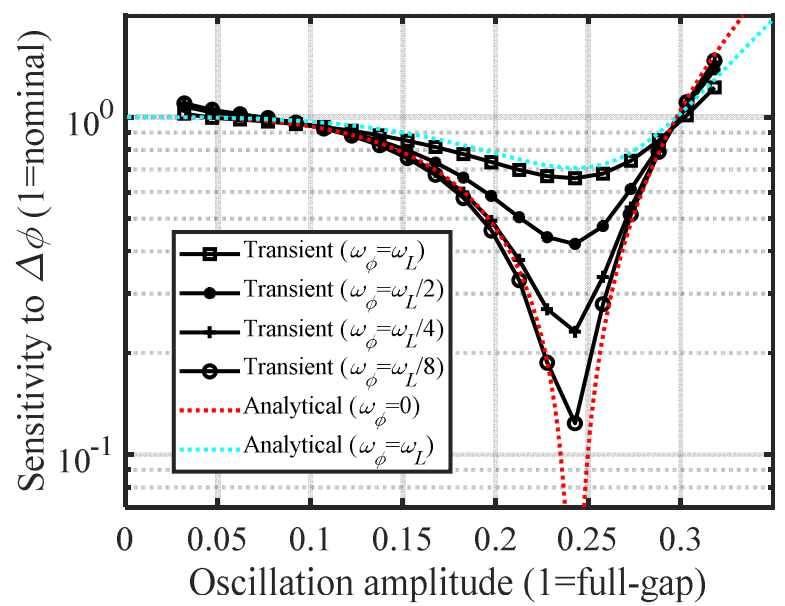

Fig. 4. Sensitivity to fluctuations of $\phi$ (normalized w.r.t. linear case). Negative pulses, $\gamma=0$.

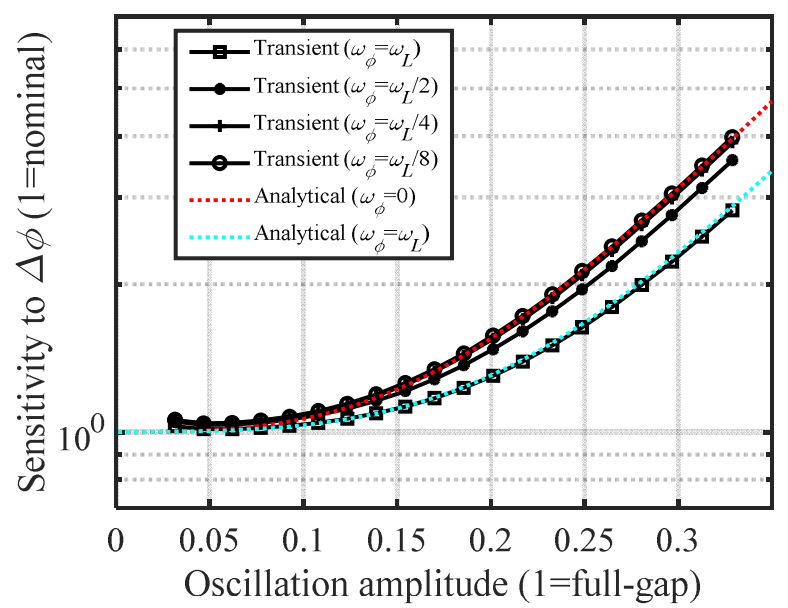

Fig. 5. Sensitivity to fluctuations of $\phi$ (normalized w.r.t. linear case). Positive pulses, $\gamma=0$.

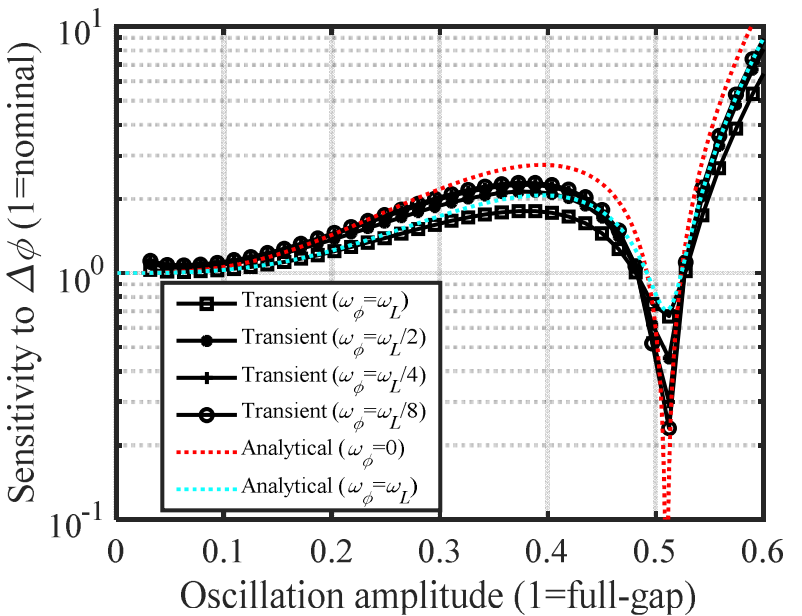

Fig. 6. Sensitivity to fluctuations of $\phi$ (normalized w.r.t. linear case). Negative pulses, $\gamma=8 \eta$.

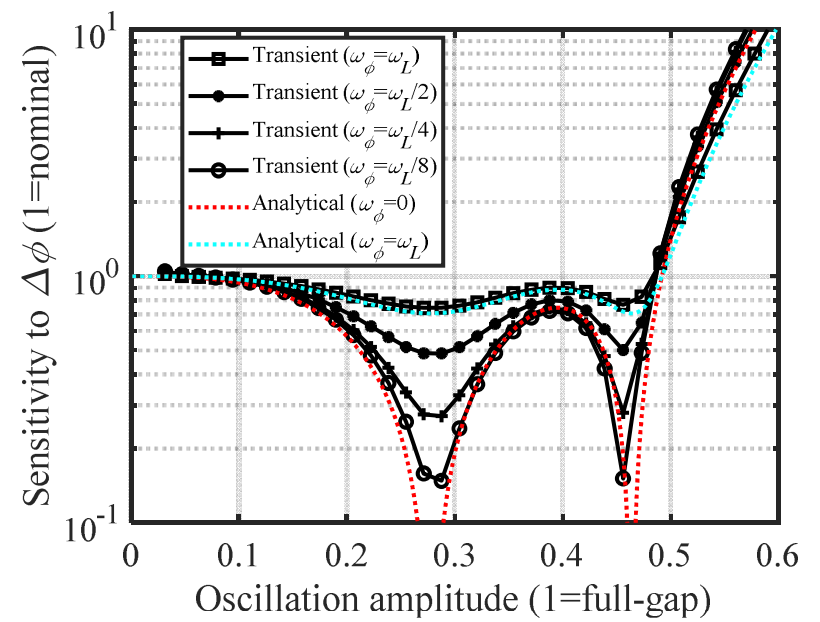

Fig. 7. Sensitivity to fluctuations of $\phi$ (normalized w.r.t. linear case). Positive pulses, $\gamma=8 \eta$.

\section{CONCLUSION AND PERSPECTIVES}

With this paper, we have proposed and validated an extension of our previous work to non-quasistatic cases. Our analytical model was validated by transient simulations (in this work) and by experiments (in [10]). This shows that parametric evasion regimes may be reached in EGCA MEMS devices without sacrificing drive efficiency, by selecting a proper driving waveform. However, as pointed out in section II, parametric evasion comes at the cost of reduced amplitude stability, which may in fact turn to have a negative impact on oscillator noise, depending on practical design.

From a more general perspective, in EGCA resonators, the amplitude and phase of all the harmonics of the driving waveform can be seen as so many design degrees of freedom, as opposed to DLA resonators where only the amplitude and phase of the first harmonic of the driving waveform matter. In particular, pulsed waveforms seem to hold a great deal of promise for EGCA resonators because of their evasion properties and their immunity to resonant pull-in, as well as having other practical advantages for all types of resonators, not covered in our system-level model. For example, subharmonic pulsed drive may be used to effectively cancel feedthrough [11], and also result in reduced switching losses and power [12]. 


\section{REFERENCES}

[1] V. Kaajakari, T. Mattila, A. Oja and H. Seppa, "Nonlinear limits for single-crystal silicon microresonators", Journal of Microelectromechanical Systems, vol. 13, pp. 715-724, 2004

[2] R. Lifshitz and M.C. Cross, "Nonlinear dynamics of nanomechanical and micromechanical resonators", Review of Nonlinear Dynamics and Complexity, Wiley, 2008

[3] D. K. Agrawal and A. A. Seshia, "An analytical formulation for phase noise in MEMS oscillators", IEEE Transactions on Ultrasonics, Ferroelectrics, and Frequency Control, vol. 61, pp. 1938-1952, 2014

[4] M. Defoort, P. Taheri-Tehrani, and D. A. Horsley, "Exploiting nonlinear amplitude-frequency dependence for temperature compensation in silicon micromechanical resonators", Applied Physics Letters, vol. 109, 153502, 2016

[5] L. G. Villanueva et al., "Surpassing fundamental limits of oscillators using nonlinear resonators", Physical Review Letters, vol. 110, 177208,2013

[6] J. Juillard and A. Brenes, "Impact of excitation waveform on the frequency stability of electrostatically-actuated microelectromechanical oscillators", Journal of Sound and Vibration, vol. 422, pp. 79-91, 2018
[7] J. Juillard, A. Brenes, M. Gouspy and M. Kraft, "Towards the optimization of excitation waveform for electrostatic resonant sensors with enhanced frequency stability", Symposium on Design, Test, Integration \& Packaging of MEMS and MOEMS (DTIP), pp. 1-4, 2019

[8] J. Juillard, "Analysis of resonant pull-in of micro-electromechanical oscillators", Journal of Sound and Vibration, vol. 350, pp. 123-139, 2015

[9] A. Brenes, J. Juillard and F. Vinci Dos Santos, "Resonant pull-in of high-Q MEMS oscillators with arbitrary closed-loop phase shift", Procedia Engineering, vol. 168, 2016, pp. 941-945

[10] J . Juillard, A. Somma, A. Brenes, "Experimental investigation of parametric evasion properties of resonant sensors using electrostatic gap-closing actuation", submitted in Sensors 2020

[11] A. Brenes, J. Juillard, F. Vinci dos Santos, A. Bonnoit, "Characterization of MEMS resonators via feedthrough de-embedding of harmonic and subharmonic pulsed-mode response", Sensors and Actuators A, vol. 229, pp.211-217, 2015

[12] L. Xu et al., "A $0.51 \mathrm{nW} 32 \mathrm{kHz}$ crystal oscillator achieving 2 ppb Allan deviation floor using high-energy-to-noise-ratio pulse injection", IEEE International Solid-State Circuits Conference, 2020. 